Article

\title{
Energy Dissipation Hypothesis Applied to Enhance the Affinity of Thrombin Binding Aptamer
}

\author{
Gleb Zhdanov ${ }^{1}$, Alexander Arutyunyuan ${ }^{2}$, Alexey Kopylov ${ }^{1} \mathbb{D}$ and Elena Zavyalova ${ }^{1, *(D)}$ \\ 1 Chemistry Department, Lomonosov Moscow State University, 119991 Moscow, Russia; \\ gleb.zhdanov@chemistry.msu.ru (G.Z.); kopylov.alex@gmail.com (A.K.) \\ 2 Belozersky Research Institute of Physical Chemical Biology, Lomonosov Moscow State University, \\ 119991 Moscow, Russia; alarut@genebee.msu.ru \\ * Correspondence: zlenka2006@gmail.com
}

Citation: Zhdanov, G.; Arutyunyuan, A.; Kopylov, A.; Zavyalova, E. Energy Dissipation Hypothesis Applied to Enhance the Affinity of Thrombin Binding Aptamer. Biophysica 2021, 1, 179-193. https://doi.org/10.3390/ biophysica1020014

Academic Editor: Danilo Milardi

Received: 20 April 2021

Accepted: 12 May 2021

Published: 14 May 2021

Publisher's Note: MDPI stays neutral with regard to jurisdictional claims in published maps and institutional affiliations.

Copyright: (c) 2021 by the authors. Licensee MDPI, Basel, Switzerland. This article is an open access article distributed under the terms and conditions of the Creative Commons Attribution (CC BY) license (https:/ / creativecommons.org/licenses/by/ $4.0 /)$.

\begin{abstract}
Nucleic acid aptamers are artificial recognizing molecules that are capable of specific binding to a wide variety of targets. Aptamers are commonly selected from a huge library of oligonucleotides and improved by introducing several mutations or modular constructions. Although aptamers hold great promise as therapeutic and diagnostic tools, no simple approach to improve their affinity has been suggested yet. Our recent analysis of aptamer-protein complexes revealed that aptamer affinity correlates with the size of an amino acid sidechain in the protein interface that was explained by efficient dissipation of the energy released during complex formation. G-quadruplexbased thrombin aptamers are not involved in the described dependence. Moreover, aptamers to the same thrombin site have 100-fold differences in affinity. Here we focused on a detailed analysis of the nucleic acid interface of thrombin-aptamer complexes. High affinity of the aptamers was shown to correlate with the solvent accessibility of the apolar part of recognizing loops. To prove the concept experimentally, these loops were modified to enhance contact with the solvent. Dissociation rates of the aptamer-thrombin complexes were drastically slowed due to these modifications. In full correspondence with the energy dissipation hypothesis, the modifications improved both the stability of the G-quadruplexes and affinity to thrombin. The most evident effect was shown for unstable $\mathrm{Na}$--coordinated G-quadruplexes. These data are of high interest for a directed improvement of aptamers introducing unnatural modifications into the 'hot spot' residues.
\end{abstract}

Keywords: aptamer; energy dissipation; modified nucleotide; protein; structure-activity relationship; thrombin

\section{Introduction}

Nucleic acid aptamers are short structured oligonucleotides that are capable of highly affine binding to a target. Commonly, aptamers are selected from huge libraries of artificial oligonucleotides based on their affinity to the target [1,2]. Point mutations, cropping of the sequence, and modular constructions are used to improve the properties of the selected aptamers during the post-selection improvement [3-5]. All of these steps are experimental, because the theory of the recognition has not been developed in detail yet.

The development of theoretical background requires an analysis of structure-activity relationships. Nearly 60 aptamer-protein complexes have been resolved to date. Analyses of the aptamer-protein structures have been performed in several works. Gelinas et al. showed that the compliance between aptamer and protein interfaces in the complexes is similar to that in antigen-antibody complexes [6]. The affinity is thought to be achieved through a set of polar contacts between the two interfaces [7,8]. The hydrophobic effect is one more significant force for aptamer-protein interaction. The affinity can be increased by slowing the rate of dissociation of the complex caused by the introduction of hydrophobic substituents into heterocycles $[9,10]$. In several cases, the hydrophobic substituents were clustered, forming a unique epitope for protein recognition $[6,10,11]$. 
Regretfully, clues to the affinity of aptamer-protein complexes mentioned above cannot be used to recalculate the affinity of the complex. It is still unclear how to predict a modification that increases the affinity by a fold. Our previous analysis of aptamer-protein interfaces revealed the absence of strict correlations between aptamer affinity and such parameters as the number of polar contacts or the interface area [12].

More thorough analysis revealed that a margin of aptamer affinity is determined by the protein interface. Bulk amino acid sidechains were associated with higher affinity compared to the smaller ones. Surprisingly, there was no correlation between the affinity and the content of positively charged or aromatic amino acids. These unexpected observations can be explained by an energy dissipation hypothesis. Namely, the bulk residues dissipate the energy released during complex formation more efficiently by decreasing the dissociation rate of the complex [13].

As for the nucleic acid interface, there is a nice example of a single modification that increased the affinity by 60 -fold. SL4 and SL 5 aptamers bind the same protein interface and have the same aptamer packing and thermal stability. The single difference is the replacement of $i$-butyl by a benzyl substituent that enlarged the number of atoms around the 'hot spot' nucleotide $[13,14]$. Here and below, the 'hot spot' residue means the residue that form three or more polar contacts in the interface. It was speculated that additional contacts of 'hot spot' residues improve the energy dissipation, increasing the stability of the aptamer-protein complex [13].

Aptamers to thrombin represent one more example where changes in aptamer affinity can be explained by the energy dissipation hypothesis. A set of the G-quadruplex-based aptamers bind the same site of thrombin; i.e., the protein interfaces are the same. However, the affinities of the aptamers differ by two-fold. The explanation was found for the aptamers with an additional duplex module. It was shown that affinity correlates with the close junction between the G-quadruplex (thrombin recognizing part) and duplex (modulating part) modules. The highest affinity was observed for the tightest junction between the two modules. It was suggested that the duplex module is engaged in the dissipation of the energy released during the aptamer-protein complex formation [15].

This paper continues a series of works aimed at elucidating the principles of the arrangement of highly affine aptamer-protein complexes. Here we analyzed the nucleic acid interface of G-quadruplex-based thrombin aptamers in detail. Aptamer modifications that can improve affinity to thrombin due to enhanced energy dissipation from the 'hot spot' nucleotides were proposed.

\section{Materials and Methods}

Inorganic salts and tris(hydroxymethyl)aminomethane (tris) were purchased from MP Biomedicals (Illkirch, France) and Sigma-Aldrich (St. Louis, MO, USA). Human thrombin with a specific activity of $4.2 \mathrm{kIU} / \mathrm{mg}$ and human factor Xa were purchased from Haematologic Technologies (Essex Junction, VT, USA). Ethanolamine from Sigma-Aldrich (St. Louis, MO, USA), 1-ethyl-3-(3-dimethylaminopropyl)- carbodiimide hydrochloride (EDC-HCl) from Roth (Karlsruhe, Germany), and sodium salt of N-hydroxysulfosuccinimide (s-NHS) from Chem-Impex Int'l (Wood Dale, IL, USA) were used. DNA oligonucleotides were synthesized and purified by polyacrylamide gel electrophoresis by Synthol (Moscow, Russia). Ultra-pure water $(18.2 \mathrm{M} \Omega \cdot \mathrm{cm}$ ) was obtained with Milli-Q (Merck KGaA, Darmstadt, Germany) and used to prepare all buffers and solutions.

\subsection{Analysis of Aptamer-Thrombin Complexes}

Structures of the aptamer-thrombin complexes were derived from Research Collaboratory for Structural Bioinformatics Protein Data Bank (RCSB PDB) [16]. The exact identification codes of the structures (pdb ids) are provided in the text. Analysis of the structures was performed using PyMol v.1.74 software (Schrodinger, New York, NY, USA). The following parameters were estimated: a number of polar contacts between the aptamer and thrombin, a list of nucleotides that form polar contacts with thrombin, a list of amino 
acids that form polar contacts with the aptamer, a list of the 'hot spot' nucleotides that form 3 or more polar contacts with thrombin, atoms of thymine (T3, T4, T12, T13) that are shielded by thrombin from the solvent, and atoms of thymines (T3, T4, T12, T13) that are in contact with the solvent. The latter two parameters were determined using the 'surface' function.

\subsection{Assembly of Aptamer Structure}

To assemble the G-quadruplex structure of the aptamers, the following algorithm was used. Aptamers were prepared in $1 \mu \mathrm{M}$ (for functional studies) or $2 \mu \mathrm{M}$ (for structural studies) concentrations in the buffer. Two buffers were used. Potassium buffer contained $10 \mathrm{mM}$ tris- $\mathrm{HCl}$ pH 7.0, $140 \mathrm{mM} \mathrm{NaCl}$, and $10 \mathrm{mM} \mathrm{KCl}$. Sodium buffer contained $10 \mathrm{mM}$ tris- $\mathrm{HCl} \mathrm{pH} 7.0$ and $140 \mathrm{mM} \mathrm{NaCl}$. The solutions were heated at $95^{\circ} \mathrm{C}$ for $5 \mathrm{~min}$ and cooled at room temperature.

\subsection{Functional Study}

Kinetic constants of association and dissociation of the aptamer-thrombin complexes were determined using the interferometer Octet Red 96 (Forte-Bio, Fremont, CA, USA). Amine reactive second-generation biosensors from Forte-Bio (Fremont, CA, USA) were used. The biosensors were hydrated for $10 \mathrm{~min}$ in sodium buffer. The solutions were placed in 96-well black plate (Greiner Bio-One, Frickenhausen, Germany) and kept at $25^{\circ} \mathrm{C}$ with mixing rate of $1000 \mathrm{rpm}$. Biosensors were activated in $200 \mathrm{mM}$ EDC and $100 \mathrm{mM}$ s-NHS for $5 \mathrm{~min}$. Then, thrombin or factor Xa (the reference protein) were immobilized from $2.5 \mu \mathrm{g} / \mathrm{mL}$ protein solutions in $40 \mathrm{mM}$ sodium phosphate buffer, $\mathrm{pH}$ 6.4. Unreacted groups were inactivated by $800 \mathrm{mM}$ ethanolamine solution, $\mathrm{pH} 8.9$ for $5 \mathrm{~min}$.

The binding experiments were performed at $25{ }^{\circ} \mathrm{C}$ with mixing rate of $1000 \mathrm{rpm}$ as follows: (1) baseline in the reaction buffer for $60 \mathrm{~s}$; (2) association stage in 25-500 nM aptamer solution for $200 \mathrm{~s}$; (3) dissociation stage in reaction buffer for $300 \mathrm{~s}$. The signal from the biosensor with the reference protein was subtracted from the signal from the biosensor with thrombin. Kinetic constants were calculated from exponential approximations of the curves [17].

\subsection{Circular Dichroism Spectroscopy}

$2 \mu \mathrm{M}$ aptamer solutions in sodium or potassium buffers were placed in quartz cuvettes with $1 \mathrm{~cm}$ path. Circular dichroism (CD) spectra were acquired using the CD spectrometer Chirascan (Applied Photophysics, Leatherhead, Great Britain) equipped with a thermoelectric temperature regulator. The spectra were acquired in the range of $200-360 \mathrm{~nm}$. The spectrum of the buffer was subtracted as a baseline. The samples were heated with a mean ramp of $1.0^{\circ} \mathrm{C} / \mathrm{min}$. Our previous experiments revealed that the melting G-quadruplex of HD1 has no hysteresis under these conditions [15]. The melting experiments were conducted in the range $5-80{ }^{\circ} \mathrm{C}$. The upper temperature limit was decreased in some cases when the intensities at $295 \mathrm{~nm}$ for 2-3 curves coincided with complete melting of the G-quadruplex. The melting temperatures were derived from the temperature dependencies of molar CD intensity at the wavelength of $295 \mathrm{~nm}$. The curves were approximated with the Boltzmann function using Origin 8.0 software (OriginLab, Northampton, MA, USA), and the parameter $\mathrm{x} 0$ corresponded to the value of the melting temperature.

\subsection{Determination of Thermodynamic Parameters}

The melting curves can be converted into thermodynamic parameters in the case of equilibrium one-step folding-unfolding processes. All calculations were provided according to Mergny and Lacroix [18]. Briefly, folded fraction $(\theta)$ can be calculated from molar circular dichroism by the Equation (1):

$$
\theta=\left(\varepsilon_{\mathrm{T}}-\varepsilon_{\mathrm{f}}\right) /\left(\varepsilon_{\mathrm{d}}-\varepsilon_{\mathrm{f}}\right)
$$


where $\varepsilon_{\mathrm{T}}$ is a molar CD at the temperature T, $\varepsilon_{\mathrm{f}}$ is a molar CD of the folded fraction, and $\varepsilon_{\mathrm{d}}$ is a molar CD of unfolded fraction. The equilibrium constant at the temperature $\mathrm{T}$ can be calculated by the Equation (2):

$$
\mathrm{K}=\theta /(1-\theta)
$$

Van't Hoff enthalpy and entropy can be derived from the temperature dependence of the equilibrium constant by plotting $\ln \mathrm{K}$ versus $1 / \mathrm{T}$ :

$$
\ln \mathrm{K}=-\Delta \mathrm{H}^{\circ} / \mathrm{RT}+\Delta \mathrm{S}^{\circ} / \mathrm{R}
$$

\section{Results}

\subsection{Analysis of Nucleic Acid Interface of Aptamers to Thrombin}

A set of X-ray complexes of the HD1-based G-quadruplex aptamers with thrombin was investigated once more. We had previously shown that the interface areas, the lists of nucleotides, the lists of atoms in polar contacts, lengths of polar contacts, and angles formed by atoms in polar contacts are nearly the same in the most affine and least affine complexes [15]. In other words, the differences in affinity cannot be explained by a sum of energies of polar contacts or the area of the interface. In this attempt, the 'hot spot' nucleotides in TT-loops were studied more thoroughly. T3, T4, T12, and T13 nucleotides of HD1 form a set of polar contacts with thrombin (Figure 1A). Two of four nucleotides were considered as the 'hot spot' nucleotides having three to four polar contacts with the protein in all X-ray structures; they were T4 and T13. In some structures, one of either the T3 or T12 nucleotides becomes an additional 'hot spot' nucleotide. Here we tested a hypothesis that the environment of these loops is different in the complexes with high and low affinity.

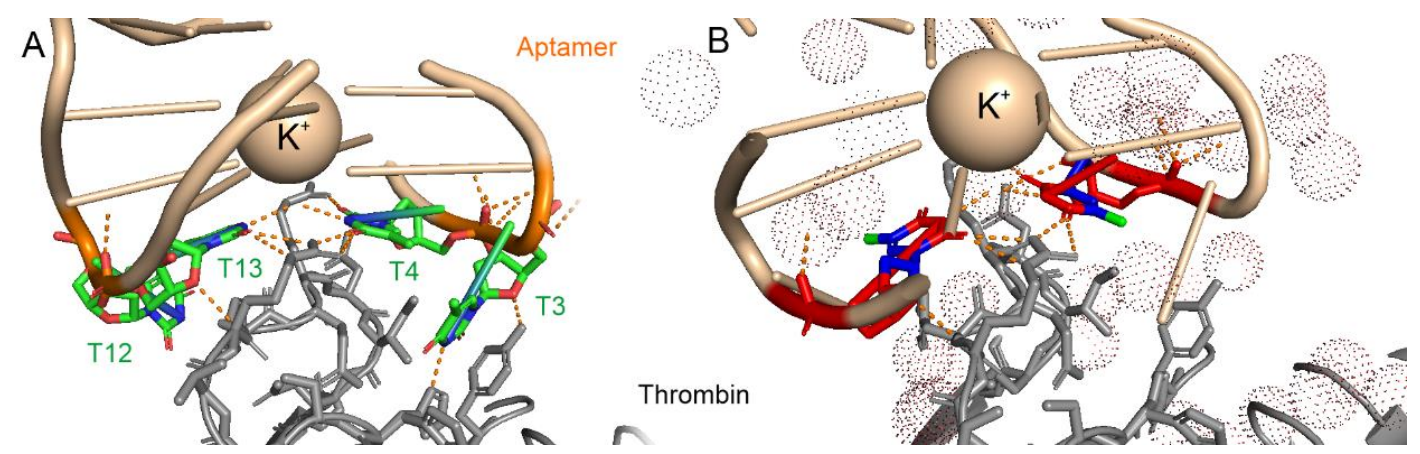

Figure 1. The interface of the HD1-thrombin complex (the structure was built from pdb id 4dii [19]); the aptamer is shown in light-brown; thrombin is shown in grey). (A) The recognizing loops are colored in green; the positions of T3, T4, T12, and T13 nucleotides are labeled; the polar contacts formed by these nucleotides are shown as dashed lines. (B) T4 and T13 are shown in color; the non-polar core is in blue and green colors; the methyl group in 5th position is shown in green. The solvent molecules are shown as transparent balls. The methyl group is faced toward the solvent; it was chosen as a site for further modifications.

The nucleotides were divided into polar groups and a non-polar core. The last comprised a set of adjacent atoms that are unable to form polar contacts, e.g., $\mathrm{C}_{\mathrm{CH} 3}, \mathrm{C} 5, \mathrm{C} 6$, $\mathrm{N} 1, \mathrm{C} 1^{\prime}$, and $\mathrm{C} 2^{\prime}$ atoms compose the non-polar core of thymidine (Figure 1B). Next, the accessibility of the non-polar core to the solvent molecules was estimated for 10 aptamerthrombin complexes (Table 1). 
Table 1. The solvent accessibility of the non-polar cores of recognizing loops of the G-quadruplexbased aptamers in the complex with thrombin.

\begin{tabular}{|c|c|c|c|c|c|c|}
\hline pdb id & $\begin{array}{l}\text { Aptamer- } \\
\text { cation }\end{array}$ & $\mathrm{K}_{\mathrm{D}}, \mathrm{nM}$ & $\begin{array}{l}\text { Atoms in } \\
\text { Contact } \\
\text { with the } \\
\text { Solvent }\end{array}$ & $\begin{array}{l}\text { Atoms } \\
\text { Shielded } \\
\text { from the } \\
\text { Solvent }\end{array}$ & $\begin{array}{c}\text { Total } \\
\text { Number } \\
\text { of Atoms }\end{array}$ & $\begin{array}{c}\text { Fraction of } \\
\text { Shielded } \\
\text { Atoms }\end{array}$ \\
\hline $41 z 4[20]$ & $\Delta \mathrm{T} 3-\mathrm{K}^{+}$ & $56[21]$ & 8 & 12 & 20 & 0.60 \\
\hline 4dih [19] & $\mathrm{HD} 1-\mathrm{Na}^{+}$ & 50 [22] & 13 & 11 & 24 & 0.46 \\
\hline $41 z 1[20]$ & $\Delta \mathrm{T} 12-\mathrm{K}^{+}$ & $42[21]$ & 9 & 11 & 20 & 0.55 \\
\hline 6gn7 [23] & NU172-Na ${ }^{+}$ & $35[24]$ & 11 & 13 & 24 & 0.54 \\
\hline $3 q l p[25]$ & mTBA-K ${ }^{+}$ & $25[26]$ & 13 & 11 & 24 & 0.46 \\
\hline 4dii [19] & HD1-K ${ }^{+}$ & $12[22]$ & 14 & 10 & 24 & 0.42 \\
\hline 6eo6 [27] & $\mathrm{T} 4 \mathrm{~W}-\mathrm{K}^{+}$ & 1 [27] & 21 & 14 & 35 & 0.40 \\
\hline $5 \mathrm{~cm} \times[28]$ & RE31-K ${ }^{+}$ & 0.56 [29] & 11 & 13 & 24 & 0.54 \\
\hline 6eo7 [27] & $\mathrm{T} 4 \mathrm{~K}-\mathrm{K}^{+}$ & 0.39 [27] & 16 & 10 & 26 & 0.38 \\
\hline 6evv [23] & NU172-K ${ }^{+}$ & 0.29 [30] & 11 & 13 & 24 & 0.54 \\
\hline
\end{tabular}

The trivial logic suggests that the non-polar core is hidden inside the interface of the complex due to the hydrophobic effect. However, the most affine complexes have the least fraction of shielded atoms in the subset of HD1-like 15-nucleotide-long aptamers coordinated by $\mathrm{K}^{+}$(Figure 2). The data of this subset can be fitted linearly with $\mathrm{R}^{2}=0.98$, which means a strong correlation. This result is the best structure-activity relationship found for thrombin aptamers. It can be explained using the energy dissipation hypothesis, i.e., the exposure of the non-polar core of the 'hot spot' nucleotides to solvent facilitates the dissipation of the energy released during complex formation. The energy dissipation proceeds from the 'hot spot' residues directly into the solvent that is the most efficient route keeping the structures of biomolecules in the folded state.

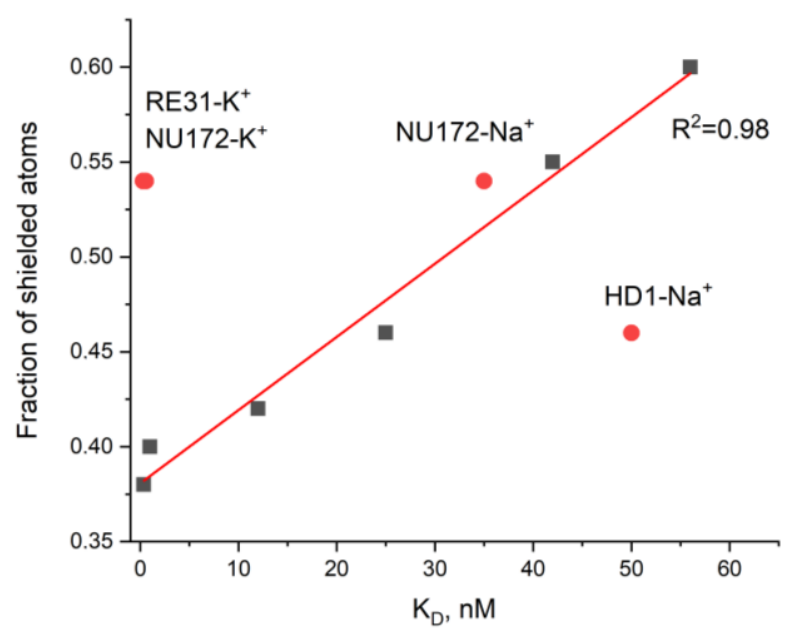

Figure 2. The correlation between dissociation constants $\left(K_{D}\right)$ and the fractions of shielded atoms in the non-polar core of the aptamer-recognizing loops. Black squares are thrombin complexes of HD1-like 15-nucleotide-long aptamers coordinated by $\mathrm{K}^{+}$; these data were fitted with a linear dependence $\left(\mathrm{R}^{2}=0.98\right)$. Other aptamer-thrombin complexes are shown in red, including two aptamers coordinated by $\mathrm{Na}^{+}$and two aptamers with the additional duplex modules.

It is interesting to consider the complexes that are out of the correlation. The complex of $\mathrm{Na}^{+}$-coordinated $\mathrm{HD} 1$ was not involved in the dependence. The most likely explanation is low stability of $\mathrm{Na}^{+}$-coordinated G-quadruplex of $\mathrm{HD} 1$ (see the experimental part below). Its melting temperature is just $23^{\circ} \mathrm{C}$; thus, nearly half of the G-quadruplexes are unfolded at room temperature, and the efficient concentration of the shaped aptamer is two times lower 
compared to $\mathrm{K}^{+}$-coordinated HD1. This peculiarity increases the equilibrium dissociation constant of the complex.

Both RE31 and NU172 are thrombin aptamers with an HD1-like G-quadruplex module and additional duplex module. The structure-activity relationship for these $\mathrm{K}^{+}$-coordinated aptamers was studied thoroughly in our previous work [15]. Both aptamers were shown to be well-packed and have rigid structures. It was suggested that the duplex module participates in the energy dissipation, keeping the G-quadruplex structure in the folded state during thrombin binding [15]. Thus, it is unsurprising for these aptamers to be out of the general trend. In contrast, $\mathrm{Na}^{+}$-coordinated NU172 lost structural rigidity, a tendency similar to that of HD1-like aptamers without the duplex modules.

\subsection{An Attempt to Experimentally Verify the Energy Dissipation Hypothesis}

The energy dissipation hypothesis can explain the changes in affinity in thrombin aptamers. It seems to be valid for the monomodular HD1-like aptamers (previous section), HD1-like aptamers with the additional duplex module [15], and generally for the aptamer-protein complexes [13]. Here we constructed a set of new HD1-like aptamers with inherently high energy dissipation ability for direct experimental verification of the hypothesis.

Both T4 and T13 are the 'hot spot' nucleotides in all HD1-like aptamer complexes with thrombin (Figure 1) [15]. Hence, these two nucleotides were chosen as a primary target for modifications. One more 'hot spot' residue is one of the T3 or T12 nucleotides that takes the T3 position in the 4dii structure [15]. The modification of T3 and T12 nucleotides was expected to have considerably less effect on the energy dissipation compared to T4 and T13 (Table 2). The non-polar core of thymines was enlarged by introducing a tail instead of the methyl group in 5th position of the cycle (Figure 3). It was expected that the size of the tail matters, whereas the introduced functional group would have a minor effect on the energy dissipation, i.e., on kinetic dissociation constants. The overall affinity changes revealed this to be more complicated.<smiles>Cc1cn(C)c(=O)[nH]c1=O</smiles>

Thymine<smiles>Cn1cc(/C=C/C(=O)[O-])c(=O)[nH]c1=O</smiles>

Carboxy-thymine<smiles>CN(C)CCCCCCNC(=O)/C=C/c1cn(C)c(=O)[nH]c1=O</smiles>

Amino-thymine<smiles>Cn1cc(/C=C/C(=O)NCCCCCCNC(=O)CCCCC2SCC3NC(=O)NC32)c(=O)[nH]c1=O</smiles>

Biotinylated thymine

Figure 3. Modifications of thymines chosen for experimental verification of the energy dissipation hypothesis.

The association and dissociation of aptamer-thrombin complexes were studied in the real-time mode. Briefly, thrombin was immobilized onto the sensor that was placed into the aptamer solution (association of the complex) and then into the empty buffer (dissociation of the complex). The experiment allowed estimations of both affinity (dissociation constant, $\left.\mathrm{K}_{\mathrm{D}}\right)$ and kinetic constants of association $\left(\mathrm{k}_{\mathrm{on}}\right)$ and dissociation $\left(\mathrm{k}_{\mathrm{off}}\right)$ of the complex. The resulting sensorgrams are provided in Figures 4 and 5. The calculated kinetic and equilibrium constants are provided in Table 3. The complexes were assembled in two buffers: (1) 'potassium buffer' was used to study folded G-quadruplex aptamers and (2) 'sodium 
buffer' was used to study sloppy G-quadruplexes that tend to be unfolded. The aptamers were assembled in the buffer used in the binding experiment.

Table 2. The sequences of HD1 and its modifications. The structures of modified nucleotides are provided in Figure 3.

\begin{tabular}{|c|c|}
\hline Aptamer & Sequence \\
\hline HD1 & $5^{\prime}$-gg-tt-gg-tgt-gg-tt-gg-3' \\
\hline carboxy-T4,T13 & $5^{\prime}$-gg-t(t-carboxy)-gg-tgt-gg-t(t-carboxy)-gg- $3^{\prime}$ \\
\hline biotin-T4,T13 & $5^{\prime}$-gg-t(t-biotin)-gg-tgt-gg-t(t-biotin)-gg- $3^{\prime}$ \\
\hline amino-T4,T13 & $5^{\prime}$-gg-t(t-amino)-gg-tgt-gg-t(t-amino $)$-gg- $3^{\prime}$ \\
\hline amino-T13 & $5^{\prime}$-gg-tt-gg-tgt-gg-t(t-amino)-gg- $3^{\prime}$ \\
\hline amino-T3,T12 & $5^{\prime}$-gg-(t-amino)t-gg-tgt-gg-(t-amino)t-gg- $3^{\prime}$ \\
\hline
\end{tabular}

Table 3. The characteristics of the aptamer-thrombin complexes, namely, dissociation constants $\left(K_{D}\right)$, kinetic association constants $\left(k_{\text {on }}\right)$, and kinetic dissociation constants $\left(k_{\text {off }}\right)$. The experiments were carried out in two buffer systems, with and without $\mathrm{K}^{+}$, that greatly affect the stability of the G-quadruplex structure. The colored cells refer to more than a 2-fold increase (green color) or decrease (orange color) in affinity. n.d.: not determinable.

\begin{tabular}{|c|c|c|c|c|c|c|}
\hline \multirow[b]{2}{*}{ Aptamer } & \multicolumn{3}{|c|}{ 'Potassium Buffer' } & \multicolumn{3}{|c|}{ 'Sodium Buffer' } \\
\hline & $\mathrm{K}_{\mathrm{D}}, \mathbf{n M}$ & $\begin{array}{c}\mathbf{k}_{\text {on, }} \\
\mu \mathbf{M}^{-1} \mathbf{s}^{-1}\end{array}$ & $\begin{array}{l}\mathrm{k}_{\mathrm{off}} \\
\mathrm{ms}^{-1}\end{array}$ & $\mathrm{~K}_{\mathrm{D}}, \mathrm{nM}$ & $\begin{array}{c}\mathbf{k}_{\mathrm{on},} \\
\mu \mathbf{M}^{-1} \mathbf{s}^{-1}\end{array}$ & $\begin{array}{l}k_{\text {off }} \\
m^{-1}\end{array}$ \\
\hline HD1 & $32 \pm 2$ & $2.7 \pm 0.4$ & $84 \pm 6$ & $180 \pm 16$ & $0.43 \pm 0.08$ & $80 \pm 20$ \\
\hline carboxy-T4,T13 & $10.1 \pm 0.6$ & $0.20 \pm 0.03$ & $2.0 \pm 0.3$ & $20.0 \pm 1.6$ & $0.19 \pm 0.03$ & $3.8 \pm 1.2$ \\
\hline biotin-T4,T13 & $19 \pm 2$ & $1.4 \pm 0.6$ & $26 \pm 4$ & $25 \pm 3$ & $0.6 \pm 0.2$ & $14 \pm 2$ \\
\hline amino-T4,T13 & $>350$ & n.d. & n.d. & $210 \pm 20$ & $0.05 \pm 0.02$ & $11.2 \pm 1.6$ \\
\hline amino-T13 & $350 \pm 40$ & $0.048 \pm 0.005$ & $16 \pm 8$ & $64 \pm 4$ & $0.25 \pm 0.04$ & $16 \pm 3$ \\
\hline amino-T3,T12 & $100 \pm 20$ & $0.8 \pm 0.4$ & $80 \pm 30$ & $77 \pm 10$ & $0.5 \pm 0.2$ & $41 \pm 12$ \\
\hline
\end{tabular}



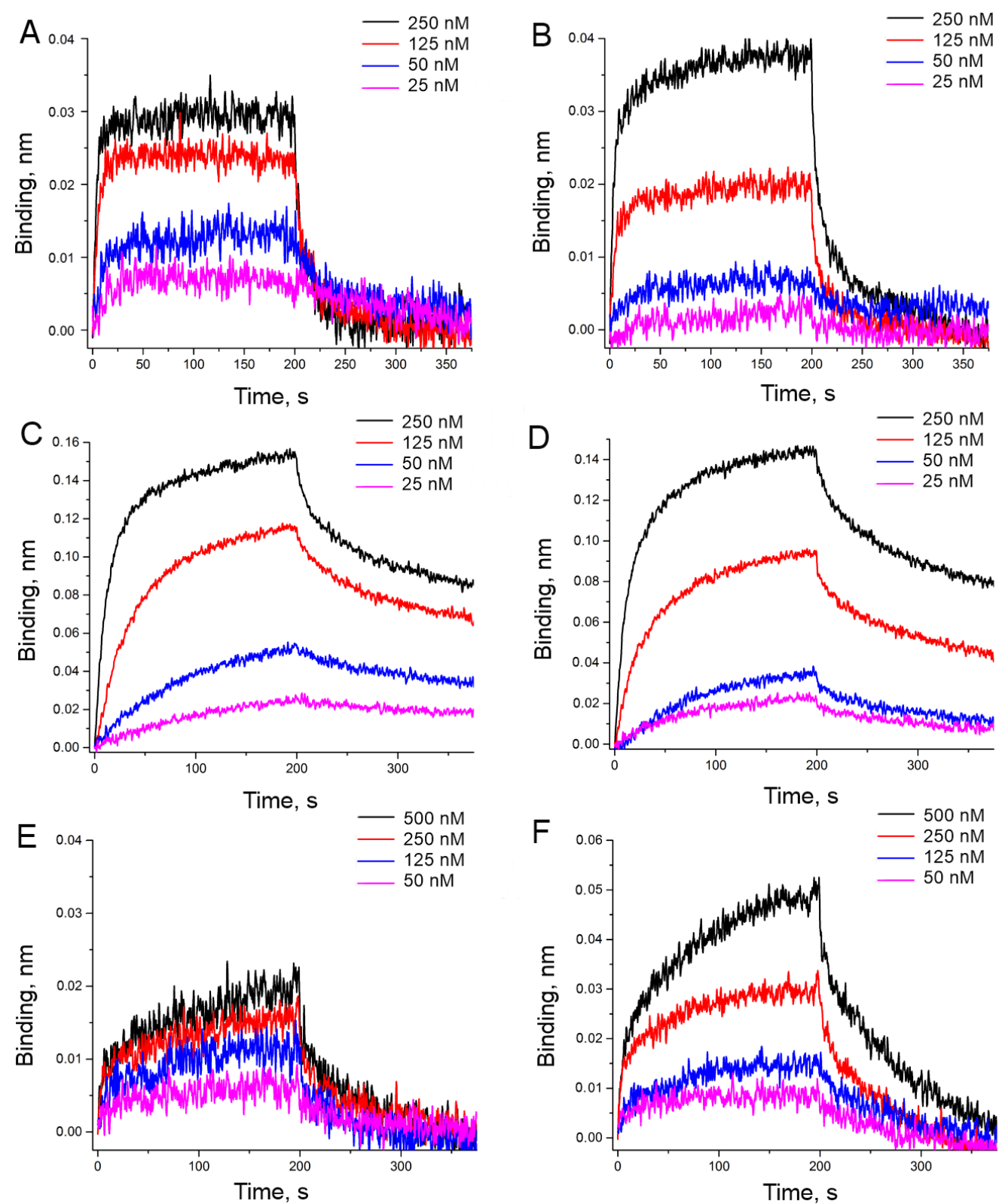

Figure 4. Binding of the aptamers to thrombin: (A,B) HD1; (C,D) carboxy-T4,T13-HD1; (E,F) biotinylated-T4,T13-HD1. The aptamer assembly and the binding experiments were carried out in 'potassium buffer' with $10 \mathrm{mM} \mathrm{K}^{+}$and $140 \mathrm{mM} \mathrm{Na}^{+}(\mathbf{A}, \mathbf{C}, \mathbf{E})$ or in 'sodium buffer' with $140 \mathrm{mM} \mathrm{Na}^{+}$ (B,D,F). The association stage was $200 \mathrm{~s}$ long; the dissociation stage was $300 \mathrm{~s}$ long. 

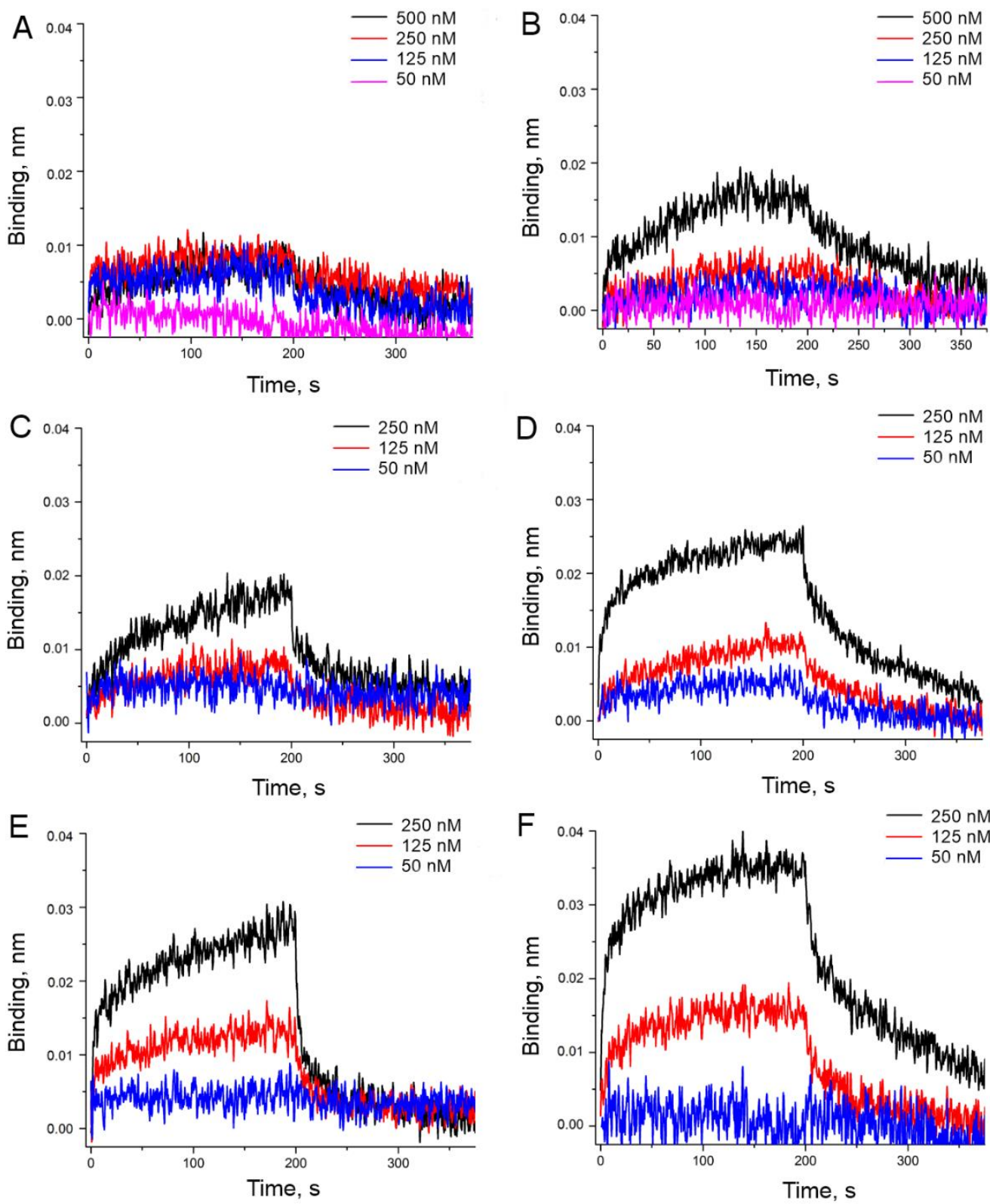

Figure 5. Binding of the amino-modified aptamers to thrombin: (A,B) amino-T4,T13-HD1; (C,D) amino-T13-HD1; (E,F) amino-T3,T12-HD1. The aptamer assembly and the binding experiments were carried out in 'potassium buffer' with $10 \mathrm{mM} \mathrm{K}^{+}$and $140 \mathrm{mM} \mathrm{Na}^{+}(\mathbf{A}, \mathbf{C}, \mathbf{E})$ or in 'sodium buffer' with $140 \mathrm{mM} \mathrm{Na}^{+}(\mathbf{B}, \mathbf{D}, \mathbf{F})$. The association stage was $200 \mathrm{~s}$ long; the dissociation stage was $300 \mathrm{~s}$ long.

The color scheme of Table 3 clearly shows the effect of the introduced modifications. Any of the suggested modifications of T4 and/or T13 nucleotides decreased the dissociation rate of the complex. This effect is in agreement with the previous theoretical findings for the protein interface of the aptamer-protein complexes [13], where the bulk amino acid residues facilitated the decrease in kinetic dissociation constant. So, that correlation is true for both the protein and nucleic acid parts of the interface. Notably, the modification of another pair of nucleotides, T3 and T12, exerted little to no effect on the complex dissociation rate, in full agreement with the expectations described above.

Interestingly, the effect of the introduced functional group became apparent at the association stage of the complex. Any charged groups slowed down the association. Positively charged groups may contribute to electrostatic repulsion from the positively charged thrombin site. The effect from the negatively charged group may be referred 
to the strong hydration coat that is to be diminished before the complexation and/or the conformation changes in the aptamer. The latter case is discussed further in the text. Uncharged biotin residue affected the association slightly, while the dissociation was slowed down 3-6 times.

The changes in the overall affinity were not so obvious, being an integral result of the two opposite trends in the association and dissociation stages. The color scheme of Table 2 clearly shows that the overall affinity was increased considerably in 'sodium buffer', i.e., in the case of sloppy G-quadruplex structures. The affinities of $\mathrm{Na}^{+}$-coordinated carboxy$\mathrm{T} 4, \mathrm{~T} 13$ and biotin-T4,T13 were even lower than the affinity of $\mathrm{K}^{+}$-coordinated HD1.

These data were unexpected. We performed additional experiments to compare the G-quadruplex state in 'sodium' and 'potassium' buffers. Circular dichroism spectroscopy is a nice tool to verify the folding of G-quadruplex, test its thermal stability, and determine the thermodynamic parameters of the structures. A set of CD spectra at different temperatures is provided in Figures 6 and 7. The melting curves were plotted for molar CD at $295 \mathrm{~nm}$ and fitted to determine the melting temperatures (Supplementary Figures S1 and S2). The resulting values are provided in Table 4 . The biotin and amino-modifications of T4 and T13 nucleotides contributed significantly to the stabilization of the G-quadruplexes. The melting temperatures were raised by $14^{\circ} \mathrm{C}$ in 'potassium' buffer and by $17^{\circ} \mathrm{C}$ in 'sodium' buffer. The antennas for energy dissipation increased the stability of both the complex with thrombin as well as the G-quadruplex structure. The sloppy $\mathrm{Na}^{+}$-coordinated Gquadruplexes became much more stable. These results are in good correlation with the results for bimodular aptamers, where an additional duplex module increased the stability of the $\mathrm{Na}^{+}$-coordinated HD1-module by $13{ }^{\circ} \mathrm{C}$ [31].

Table 4. The structural characteristics of the aptamers in two buffer systems. The data derived from circular dichroism experiments: melting temperatures $\left(\mathrm{T}_{\mathrm{m}}\right)$, enthalpy changes $\left(\Delta \mathrm{H}^{\circ}\right)$, entropy changes $\left(\Delta \mathrm{S}^{\circ}\right)$, and Gibbs free energy changes $\left(\Delta \mathrm{G}^{\circ}{ }_{298}\right)$ during $\mathrm{G}$-quadruplex folding. The data for the aptamers with melting temperature increases of $10{ }^{\circ} \mathrm{C}$ and more are highlighted in green.

\begin{tabular}{|c|c|c|c|c|c|c|c|c|}
\hline \multirow[b]{2}{*}{ Aptamer } & \multicolumn{4}{|c|}{ ‘Potassium Buffer' } & \multicolumn{4}{|c|}{ 'Sodium Buffer' } \\
\hline & $\mathrm{T}_{\mathrm{m}},{ }^{\circ} \mathrm{C}$ & $\begin{array}{c}\Delta \mathrm{H}^{\circ}, \\
\mathrm{kJ} / \mathrm{mol}\end{array}$ & $\Delta \mathrm{S}^{\circ}, \mathrm{J} / \mathrm{mol}$ & $\begin{array}{l}\Delta \mathrm{G}^{\circ} 298 \\
\mathrm{~kJ} / \mathrm{mol}\end{array}$ & $\mathrm{T}_{\mathrm{m}},{ }^{\circ} \mathrm{C}$ & $\begin{array}{c}\Delta \mathrm{H}^{\circ} \\
\mathrm{kJ} / \mathrm{mol}\end{array}$ & $\Delta \mathrm{S}^{\circ}, \mathrm{J} / \mathrm{mol}$ & $\begin{array}{l}\Delta \mathrm{G}^{\circ} 298 \\
\mathrm{~kJ} / \mathrm{mol}\end{array}$ \\
\hline HD1 & $38.7 \pm 0.1$ & $-151 \pm 3$ & $-485 \pm 9$ & $-6 \pm 2$ & $22.8 \pm 0.6$ & $-131 \pm 4$ & $-443 \pm 12$ & $1 \pm 3$ \\
\hline carboxy-T4,T13 & $40.3 \pm 0.6$ & $-77 \pm 2$ & $-245 \pm 5$ & $-3.5 \pm 1.1$ & $34.0 \pm 0.5$ & $-97 \pm 2$ & $-315 \pm 8$ & $-2.8 \pm 1.6$ \\
\hline biotin-T4,T13 & $52.6 \pm 0.4$ & $-136 \pm 5$ & $-417 \pm 14$ & $-11 \pm 3$ & $39.5 \pm 0.3$ & $-109 \pm 2$ & $-351 \pm 7$ & $-4.8 \pm 1.5$ \\
\hline amino-T4,T13 & $52.6 \pm 0.5$ & $-120 \pm 5$ & $-369 \pm 17$ & $-10 \pm 4$ & $39.7 \pm 0.4$ & $-123 \pm 2$ & $-395 \pm 7$ & $-5.6 \pm 1.7$ \\
\hline amino-T13 & $46.0 \pm 0.3$ & $-121 \pm 3$ & $-382 \pm 9$ & $-8 \pm 2$ & $30.1 \pm 0.7$ & $-113 \pm 3$ & $-373 \pm 10$ & $-2 \pm 2$ \\
\hline amino-T3,T12 & $40.6 \pm 0.2$ & $-147 \pm 3$ & $-469 \pm 9$ & $-7 \pm 2$ & $25.8 \pm 0.2$ & $-118 \pm 2$ & $-394 \pm 6$ & $-0.3 \pm 1.3$ \\
\hline
\end{tabular}



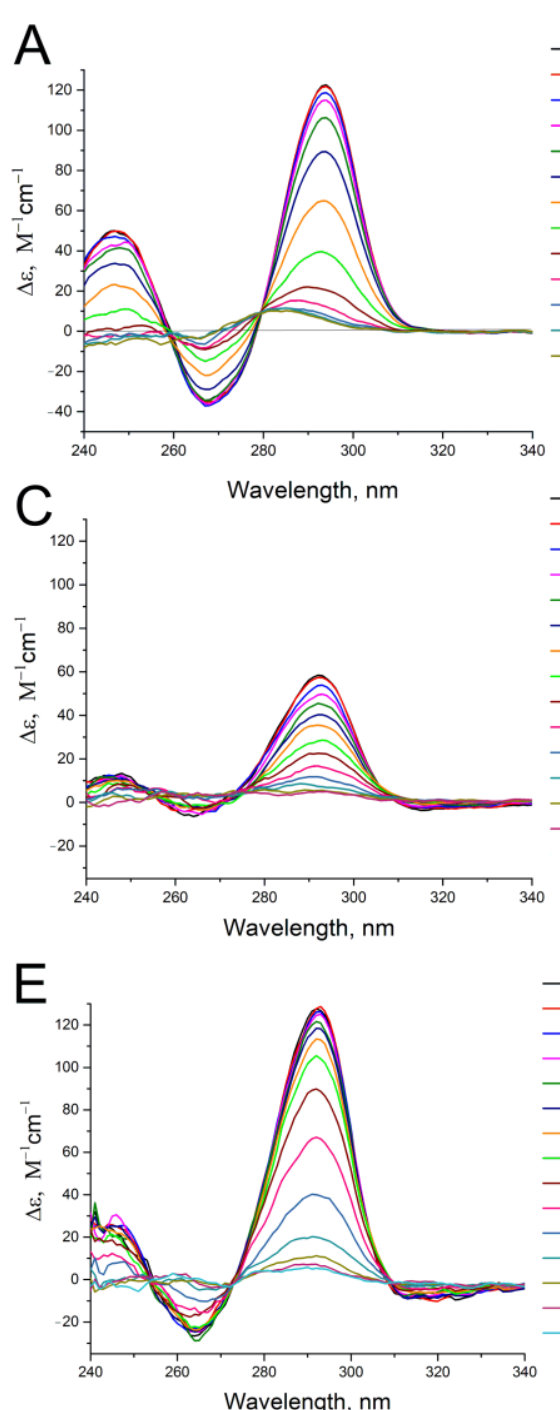

B

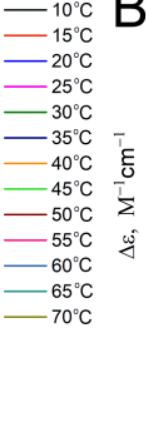

- $100 \mathrm{C}$
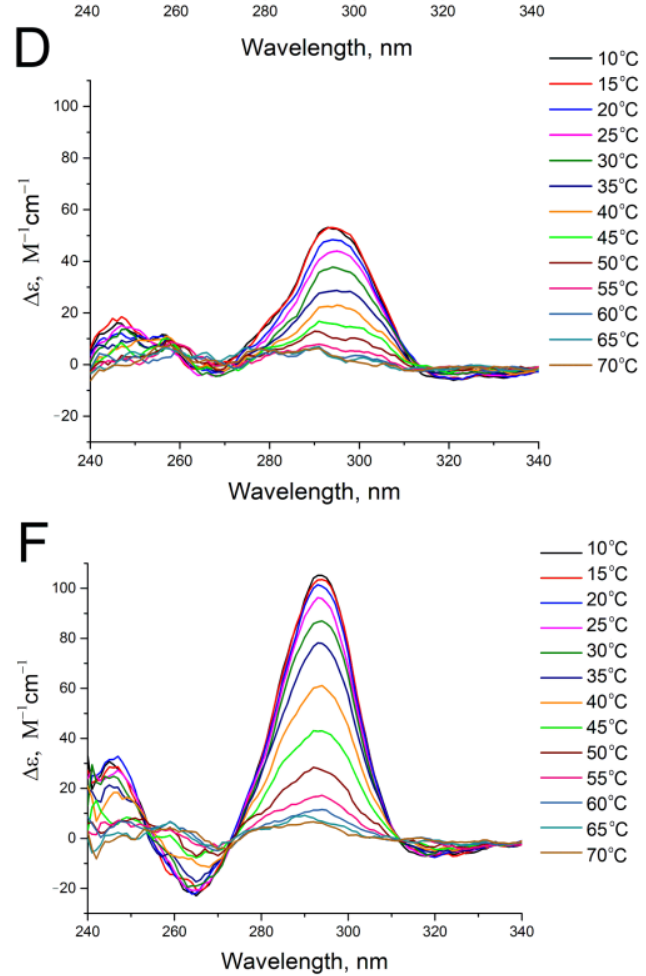

Figure 6. Circular dichroism spectra obtained at different temperatures for the aptamers: (A) $\mathrm{K}^{+}$coordinated HD1; (B) $\mathrm{Na}^{+}$-coordinated HD1; (C) $\mathrm{K}^{+}$-coordinated carboxy-T4,T13-HD1; (D) $\mathrm{Na}^{+}$coordinated carboxy-T4,T13-HD1; (E) $\mathrm{K}^{+}$-coordinated biotinylated-T4,T13-HD1; (F) $\mathrm{Na}^{+}$-coordinated biotinylated-T4,T13-HD1. 
A

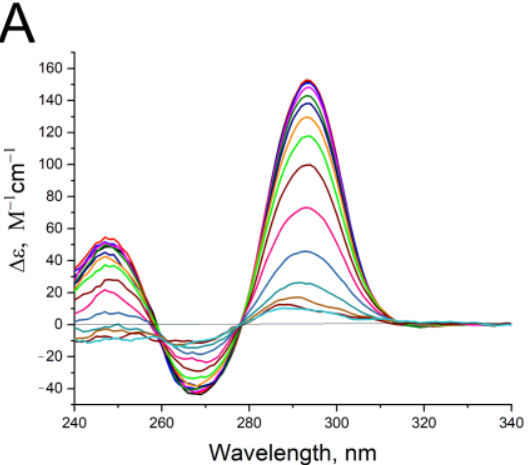

$\mathrm{C}$

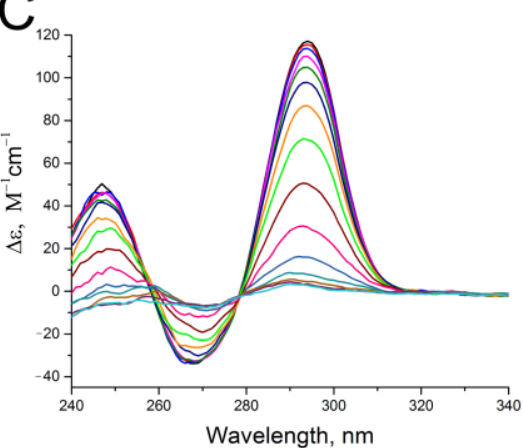

E

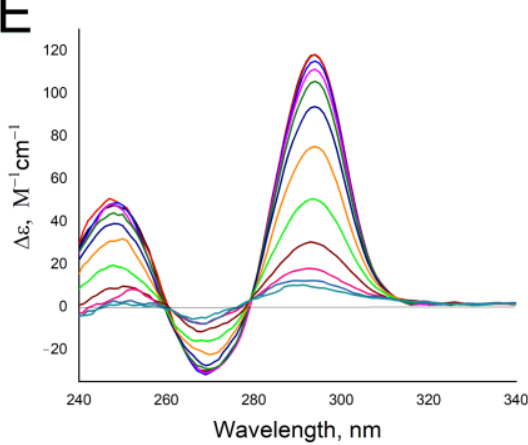

B
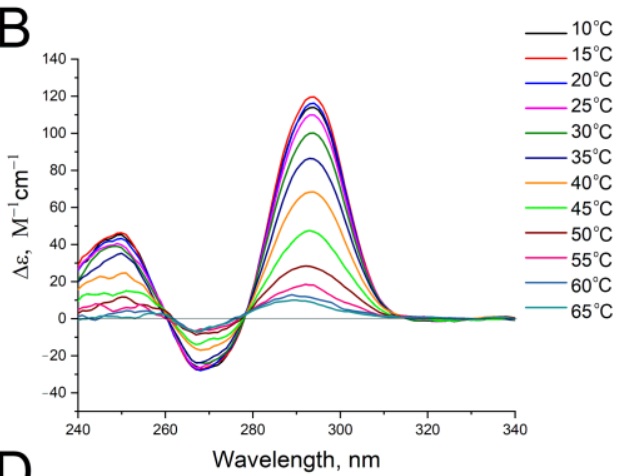

$\mathrm{D}$
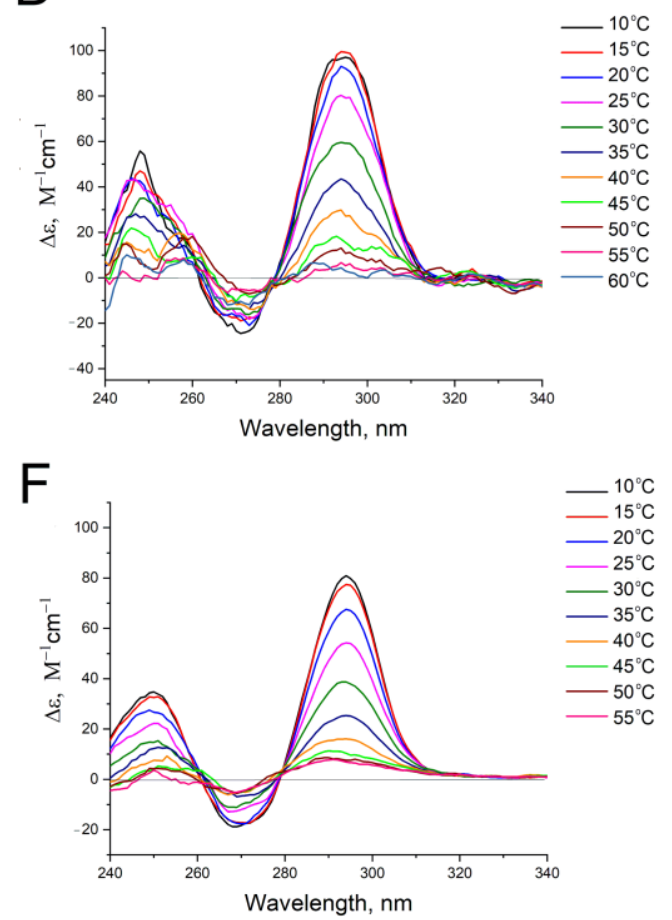

Figure 7. Circular dichroism spectra obtained at different temperatures for the aptamers: (A) $\mathrm{K}^{+}$coordinated amino-T4,T13-HD1; (B) $\mathrm{Na}^{+}$-coordinated amino-T4,T13-HD1; (C) $\mathrm{K}^{+}$-coordinated aminoT13-HD1; (D) Na ${ }^{+}$-coordinated amino-T13-HD1; (E) $\mathrm{K}^{+}$-coordinated amino-T3,T12-HD1; (F) $\mathrm{Na}^{+}$coordinated amino-T3,T12-HD1.

The thermodynamic characteristics were derived from further calculations (Supplementary Figures S3 and S4, Table 4). All the modifications decreased the absolute values of enthalpy and entropy changes. As a result of two opposite events, the absolute values of the Gibbs free energy changes were increased compared to the initial HD1 aptamer with a sole exception. Carboxy-T4,T13, became less stable in the 'potassium' buffer; its CD spectra (Figure 6C,D) reveal a distorted antiparallel G-quadruplex that can be explained by the increase of the dipoles in T4 and T13 due to the mesomeric effect of the conjugated carboxyl.

As the trends for Gibbs free energy and entropy changes coincided, it could be summarized that the aptamer stabilization is entropy-driven.

A set of thermodynamic parameters was used to find a correlation with equilibrium or kinetic constants for aptamer-thrombin complexes. The correlation was found only between kinetic dissociation constants and changes in the entropy or enthalpy of the Gquadruplexes (Figure 8). This means that the rigid and well-structured G-quadruplexes dissociated more readily from the complex with thrombin. 

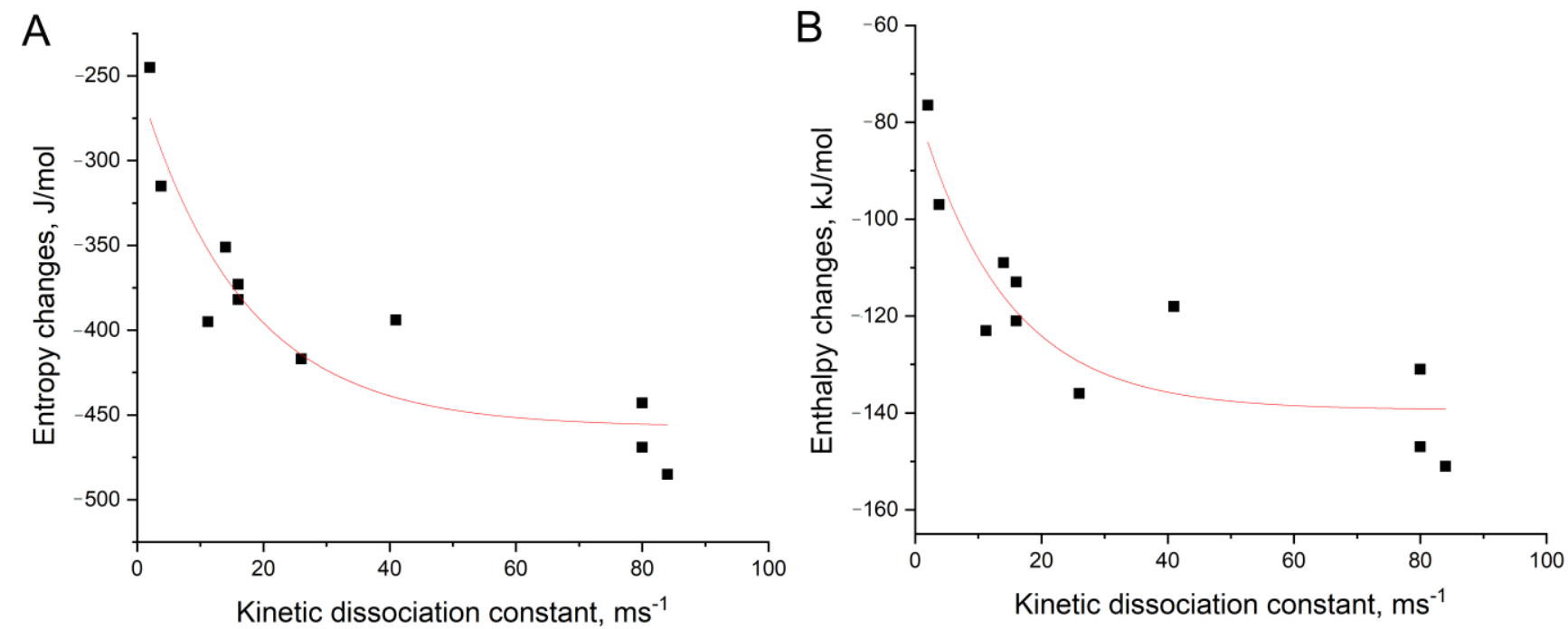

Figure 8. The relationship between thermodynamic parameters of G-quadruplex and characteristics of aptamer-thrombin complexes. (A) a correlation between the kinetic dissociation constant and the entropy change of G-quadruplex folding. (B) a correlation between the kinetic dissociation constant and the enthalpy change of the G-quadruplex folding.

\section{Conclusions}

The energy dissipation hypothesis derived from the theoretical analysis was proved experimentally for the thrombin aptamers. The introduction of the solvent-accessible antennas into the 'hot spot' residues is a good approach to slowing down the dissociation of the complex. Additionally, these modifications increased the thermal stability of the G-quadruplex due to significant changes in the entropic summand. The idea of efficient energy dissipation as one of the key parameters of highly affine complexes was proven both in the case of site-specific introduction of the antennas and bimodular aptamers [15], where high affinity is associated with the perfect stacking with duplex modules [15,31].

Supplementary Materials: The following are available online at https:/ / www.mdpi.com/article/ 10.3390/biophysica1020014/s1, Figure S1: Melting curves of G-quadruplexes acquired from circular dichroism spectra at wavelength of 295 nm; Figure S2: Melting curves of G-quadruplexes acquired from circular dichroism spectra at wavelength of $295 \mathrm{~nm}$; Figure S3: Linearization of melting curves in the coordinates $\ln \mathrm{K}(1 / \mathrm{T})$ used for the calculations of thermodynamic parameters; Figure S4: Linearization of melting curves in the coordinates $\ln K(1 / \mathrm{T})$ used for the calculations of thermodynamic parameters.

Author Contributions: Conceptualization, E.Z.; methodology, A.A. and A.K.; formal analysis, G.Z. and E.Z.; investigation, G.Z. and E.Z.; writing—original draft preparation, E.Z.; writing-review and editing, G.Z. and E.Z.; visualization, E.Z.; supervision, E.Z.; project administration, E.Z.; funding acquisition, E.Z. All authors have read and agreed to the published version of the manuscript.

Funding: This research was funded by Council for Grants of the President of the Russian Federation, grant number SP-1680.2018.4.

Data Availability Statement: The data are not available in free access, but can be provided on the request.

Conflicts of Interest: The authors declare no conflict of interest.

\section{References}

1. Blind, M.; Blank, M. Aptamer selection technology and recent advances. Mol. Ther. Nucleic Acids 2015, 4, e223. [CrossRef]

2. Zhang, Y.; Lai, B.S.; Juhas, M. Recent advances in aptamer discovery and applications. Molecules 2019, 24, 941. [CrossRef]

3. Kalra, P.; Dhiman, A.; Cho, W.C.; Bruno, J.G.; Sharma, T.K. Simple methods and rational design for enhancing aptamer sensitivity and specificity. Front. Mol. Biosci. 2018, 5, 41. [CrossRef] 
4. Elskens, J.P.; Elskens, J.M.; Madder, A. Chemical modification of aptamers for increased binding affinity in diagnostic applications: Current status and future prospects. Int. J. Mol. Sci. 2020, 21, 4522. [CrossRef]

5. Hasegawa, H.; Savory, N.; Abe, K.; Ikebukuro, K. Methods for improving aptamer binding affinity. Molecules 2016, $21,421$. [CrossRef] [PubMed]

6. Gelinas, A.; Davies, D.; Janjic, N. Embracing proteins: Structural themes in aptamer-protein complexes. Curr. Opin. Struct. Biol. 2016, 36, 122-132. [CrossRef]

7. Yatime, L.; Maasch, C.; Hoehlig, K.; Klussmann, S.; Andersen, G.R.; Vater, A. Structural basis for the targeting of complement anaphylatoxin C5a using a mixed L-RNA/L-DNA aptamer. Nat. Commun. 2015, 6, 6481. [CrossRef] [PubMed]

8. Huang, R.H.; Fremont, D.H.; Diener, J.L.; Schaub, R.G.; Sadler, J.E. A structural explanation for the antithrombotic activity of ARC1172, a DNA aptamer that binds von Willebrand factor domain A1. Structure 2009, 17, 1476-1484. [CrossRef] [PubMed]

9. Kato, K.; Ikeda, H.; Miyakawa, S.; Futakawa, S.; Nonaka, Y.; Fujiwara, M.; Okudaira, S.; Kano, K.; Aoki, J.; Morita, J.; et al. Structural basis for specific inhibition of Autotaxin by a DNA aptamer. Nat. Struct. Mol. Biol. 2016, 23, 395-401. [CrossRef] [PubMed]

10. Ren, X.; Gelinas, A.D.; von Carlowitz, I.; Janjic, N.; Pyle, A.M. Structural basis for IL-1 $\alpha$ recognition by a modified DNA aptamer that specifically inhibits IL-1 $\alpha$ signaling. Nat. Commun. 2017, 8, 810. [CrossRef]

11. Jarvis, T.C.; Davies, D.R.; Hisaminato, A.; Resnicow, D.I.; Gupta, S.; Waugh, S.M.; Nagabukuro, A.; Wadatsu, T.; Hishigaki, H.; Gawande, B.; et al. Non-helical DNA triplex forms a unique aptamer scaffold for high affinity recognition of nerve growth factor. Structure 2015, 23, 1293-1304. [CrossRef]

12. Novoseltseva, A.; Zavyalova, E.; Golovin, A.; Kopylov, A. An insight into aptamer-protein complexes. Aptamers 2018,2 , 1-19.

13. Zavyalova, E.; Kopylov, A. Energy transfer as a driving force in nucleic acid-protein interactions. Molecules 2019, $24,1443$. [CrossRef] [PubMed]

14. Davies, D.R.; Gelinas, A.D.; Zhang, C.; Rohloff, J.C.; Carter, J.D.; O'Connell, D.; Waugh, S.M.; Wolk, S.K.; Mayfield, W.S.; Burgin, A.B.; et al. Unique motifs and hydrophobic interactions shape the binding of modified DNA ligands to protein targets. Proc. Natl. Acad. Sci. USA 2012, 109, 19971-19976. [CrossRef] [PubMed]

15. Zavyalova, E.G.; Legatova, V.A.; Alieva, R.S.; Zalevsky, A.O.; Tashlitsky, V.N.; Arutyunyan, A.M.; Kopylov, A.M. Putative mechanisms underlying high inhibitory activities of bimodular DNA aptamers to thrombin. Biomolecules 2019, 9, 41. [CrossRef] [PubMed]

16. Research Collaboratory for Structural Bioinformatics Protein Data Bank (RCSB PDB). Available online: https://www.rcsb.org/ (accessed on 15 March 2021).

17. SPR-Pages. Available online: https://www.sprpages.nl/ (accessed on 15 March 2021).

18. Mergny, J.L.; Lacroix, L. Analysis of thermal melting curves. Oligonucleotides 2003, 13, 515-537. [CrossRef]

19. Russo Krauss, I.; Merlino, A.; Randazzo, A.; Novellino, E.; Mazzarella, L.; Sica, F. High-resolution structures of two complexes between thrombin and thrombin-binding aptamer shed light on the role of cations in the aptamer inhibitory activity. Nucleic Acids Res. 2012, 40, 8119-8128. [CrossRef]

20. Pica, A.; Russo Krauss, I.; Merlino, A.; Nagatoishi, S.; Sugimoto, N.; Sica, F. Dissecting the contribution of thrombin exosite I in the recognition of thrombin binding aptamer. FEBS J. 2013, 280, 6581-6588. [CrossRef]

21. Nagatoishi, S.; Sugimoto, N. Interaction of water with the G-quadruplex loop contributes to the binding energy of G-quadruplex to protein. Mol. Biosyst. 2012, 8, 2766-2770. [CrossRef]

22. Zavyalova, E.; Kopylov, A. G-quadruplexes and i-motifs as scaffolds for molecular engineering of DNA aptamers. In G-Quadruplex Structures, Formation and Roles in Biology; Santos, H., Ed.; Nova Publishers: New York, NY, USA, 2016; pp. 53-80.

23. Troisi, R.; Napolitano, V.; Spiridonova, V.; Russo Krauss, I.; Sica, F. Several structural motifs cooperate in determining the highly effective anti-thrombin activity of NU172 aptamer. Nucleic Acids Res. 2018, 46, 12177-12185. [CrossRef]

24. Zavyalova, E.; Tagiltsev, G.; Reshetnikov, R.; Arutyunyan, A.; Kopylov, A. Cation coordination alters the conformation of a thrombin-binding G-quadruplex DNA aptamer that affects inhibition of thrombin. Nucleic Acid Ther. 2016, 26, 299-308. [CrossRef]

25. Russo Krauss, I.; Merlino, A.; Giancola, C.; Randazzo, A.; Mazzarella, L.; Sica, F. Thrombin-aptamer recognition: A revealed ambiguity. Nucleic Acids Res. 2011, 39, 7858-7867. [CrossRef] [PubMed]

26. Pagano, B.; Martino, L.; Randazzo, A.; Giancola, C. Stability and binding properties of a modified thrombin binding aptamer. Biophys. J. 2008, 94, 562-569. [CrossRef]

27. Dolot, R.; Lam, C.H.; Sierant, M.; Zhao, Q.; Liu, F.W.; Nawrot, B.; Egli, M.; Yang, X. Crystal structures of thrombin in complex with chemically modified thrombin DNA aptamers reveal the origins of enhanced affinity. Nucleic Acids Res. 2018, 46, 4819-4830. [CrossRef] [PubMed]

28. Russo Krauss, I.; Spiridonova, V.; Pica, A.; Napolitano, V.; Sica, F. Different duplex/quadruplex junctions determine the properties of anti-thrombin aptamers with mixed folding. Nucleic Acids Res. 2016, 44, 983-991. [CrossRef]

29. Spiridonova, V.A.; Barinova, K.V.; Glinkina, K.A.; Melnichuk, A.V.; Gainutdynov, A.A.; Safenkova, I.V.; Dzantiev, B.B. A family of DNA aptamers with varied duplex region length that forms complexes with thrombin and prothrombin. FEBS Lett. 2015, 589, 2043-2049. [CrossRef] [PubMed] 
30. Zavyalova, E.; Golovin, A.; Pavlova, G.; Kopylov, A. Module-activity relationship of G-quadruplex based DNA aptamers for human thrombin. Curr. Med. Chem. 2013, 20, 4836-4843. [CrossRef] [PubMed]

31. Russo Krauss, I.; Napolitano, V.; Petraccone, L.; Troisi, R.; Spiridonova, V.; Mattia, C.A.; Sica, F. Duplex/quadruplex oligonucleotides: Role of the duplex domain in the stabilization of a new generation of highly effective anti-thrombin aptamers. Int. J. Biol. Macromol. 2018, 107, 1697-1705. [CrossRef] 